\title{
Development of a Method of Diagnosing Ovarian Disorders in Sows and Gilts Using Uterine Ultrasonography
}

\author{
Hiroshi TORIUMI ${ }^{1}$, Shigehisa TSUMAGARI ${ }^{1)}$, Yasushi KUWAHARA ${ }^{1)}$, Yasuaki ICHIKAWA ${ }^{1)}$, Masatoshi TAKEISHI ${ }^{1)}$ \\ and Takeo SAKAI ${ }^{2)}$
}

${ }^{1)}$ Departments of Theriogenology ${ }^{2)}$ Preventive Veterinary Medicine and Animal Health, College of Bioresource Sciences, Nihon

University, Fujisawa, Kanagawa 242-8510, Japan

(Received 27 March 2001/Accepted 12 November 2002)

ABSTRACT. In ultrasonographic diagnosis of ovarian disorders and the estrous cycle in sows, transverse observation of the uterus yielded
more characteristic findings than observation of sagittal sections. Transverse ultrasonography revealed that the low progesterone (P) type
uterus showed a round structure, while the high P type uterus showed a flattened structure. These results corresponded well with rectal
palpation findings: the low P type uterus had a hard, pipe-like structure and the high P type a soft, balloon-like structure. For gilts, we
employed a minimum convex type transrectal prostate probe that had an approximately $18 \mathrm{~cm}$ insertion handle. The images of the uterus
obtained thereby were a similar to those obtained from sows. The above results suggest that it should be possible to diagnose and treat
many ovarian disorders in sows and gilts based only on the ultrasonograhic findings. In short, ultrasonograhic findings of a round struc-
ture of the uterine wall might be an indication for PMSG (pregnant mare serum gonadotropin) treatment, while findings of a flattened
structure might be an indication for $\mathrm{PGF}_{2 \alpha}$ administration. KEY WORDS: ovarian disorder, rectal palpation, swine, ultrasonography.

In sows, the body size is relatively small and the pelvic cavity is narrow. Therefore, rectal examination is difficult to perform and requires a high level of technical skill in order to diagnose ovarian disorders. Especially, in gilts, rectal palpation is almost impossible due to the small body and pelvic size. Presently, most sows with ovarian disorders are culled without exact diagnosis or therapy.

Transcutaneous ultrasonic imaging is used in sows mostly for early detection of pregnancy $[2,3,6,11]$, while transrectal ultrasound imaging is mostly used to diagnose ovarian and uterine disorders, and to detect ovulation $[8,10$, $12,13]$. However, it is more difficult to manually operate a probe in the rectum than to perform rectal palpation, so there have been very few reports describing the use of ultrasonic imaging to diagnose ovarian disorders in sows [8].

The uterine shape as determined by rectal palpation in sows with postweaning ovarian disorders is classifiable into two types based on the serum progesterone $(\mathrm{P})$ concentration: a cylindrical pipe-like structure associated with a low serum $\mathrm{P}$ concentration or a wide balloon-like structure associated with a high serum $P$ concentration [17]. The pipe-like uterus also feels hard and the balloon-like uterus feels soft on rectal palpation. Therefore, the authors proposed a new theory of rectal palpation based on uterine shape that may reduce the rate of misdiagnoses and provide more exact information than the usual rectal examination $[14,17]$. However, it may be considered that it would be difficult to reproduce rectal palpation findings by practitioners who have not yet mastered rectal examination in sows. In this study authors compared findings obtained from rectal palpation and findings on transrectal ultrasonography and explored the feasibility of performing transrectal ultrasonography in gilts without insertion of the palm using a transrectal prostate probe, which has an approximately 18 $\mathrm{cm}$ insertion handle.

\section{MATERIALS AND METHODS}

Sows: The sows used in this study were obtained from a breeding farm in Kanagawa Prefecture in Japan. We used 5 gilts and 5 postweaning sows with a regular estrous cycle and 42 postweaning sows with ovarian disorders selected from 216 sows that were anestrus for more than 7 days after weaning and were diagnosed with ovarian disorders by rectal examination. The ovarian disorders in 42 sows consisted of 10 cases of ovarian atrophy (OA), 10 cases of ovarian quiescence (OQ), 5 cases of multiple large follicular cysts (MLFC), 2 cases of multiple small follicular cysts (MSFC), 5 cases of multiple large luteal cysts (MLLC) and 10 cases of retention of corpora lutea (RCL). All sows were 2-4 years of age, and none received medical treatment during the study. The first examination was performed at the request of the swine farmers because sows did not show signs of estrus for over 7 days after the postweaning anestrous period. Rectal examination and ultrasonic imaging were performed at 7-day intervals an additional 2 to 3 times over a 21-day period.

Ultrasonography of sows with normal estrous cycle: First, we performed rectal palpation in sows with a normal estrous cycle, and the estrous (period of sexual receptivity) and luteal phases (12 days after ovulation) were identified. Following rectal palpation, differences in sagittal and transverse sectional ultrasonography were compared.

Ultrasonography of sows with ovarian disorders: Secondly, we performed rectal palpation in sows with ovarian disorders and diagnosed the diseases by the method 
described below. Following rectal palpation, differences in sagittal and transverse sectional ultrasonography were compared. Ovarian disorders were diagnosed based on the Guidelines for Diagnosis and Therapy for Livestock in Japan [9], and the reports of Torium et al. [17] and Estill et al. [5] using the following diagnostic standards.

Ovarian subfunction: a) OQ: Sows are without obvious signs of estrus even though the postweaning anestrous period should be finished. Ovaries are nearly normal in size, and small follicles exist but are not developed or are developed but not ovulated, and there are multiple atretic corpora lutea. The uterus is small, has a pipe-like shape and is inflexible. b) OA: Sows are without obvious signs of estrus even though the postweaning anestrous period should be finished. The ovaries are small, atrophied and indurate, approximately the size of the tip of a person's small finger, and contain no obvious follicles or corpora lutea. The uterus is very thin with pipe-like shape and inflexible.

$M L F C$ : The ovaries are extremely large and located in deeper positions in the abdominal cavity than in normal animals. Large cysts 15 to $50 \mathrm{~mm}$ in diameter are observed in considerable numbers in the ovaries, but can not be detected in the normal corpora lutea. The walls of these cysts are extremely thin and easily ruptured by finger pressure. During the study period, the ovaries do not show remarkable changes. The uteri are mostly large with a pipe-like shape, and have no contractions.

$M S F C$ : Sows continuously show estrous signs without ovulation. A considerable number of small follicular cysts with diameters less than $8 \mathrm{~mm}$ are palpabled in the ovaries. These follicular cysts are smaller than mature follicles ( 8 to $10 \mathrm{~mm}$ in diameter) of sows with normal estrus, and the walls of the cysts are slightly thickened. There is no sign of ovulation and ther are no corpora lutea. The uteri typically have a pipe-like shape and medium to high contractility.

$R C L$ : The ovaries are situated in the usual position in the abdominal cavity and are soft and elastic. Numerous corpora lutea 8 to $12 \mathrm{~mm}$ in diameter are observed. Small follicles coexist with these corpora lutea. The size and location of the corpora lutea do not change over a period of 10 to 14 days. Although the uteri are balloon-like in shape, they are not loose and appear similar to uteri in the normal luteal phase.

$M L L C$ : The ovaries are extremely large and slightly tender and soft, but elastic, and there are no normal corpora lutea are detected. Numerous cysts 15 to $50 \mathrm{~mm}$ in diameter are observed. These cysts have thickened, elastic, strong walls that are not ruptured easily by finger pressure. During the experimental period, the thickness and elasticity of the cyst wall increases with time. The uterus, dropping in the abdominal cavity, is balloon-like in structure, soft, thinwalled and loose. The uterine palpation findings are clearly different from those in other disorders.

Equipment: An ultrasonic imaging device (EUB-905 from Hitachi Medico (Tokyo, Japan)) was used. The probes employed were a 7.5 MHz convex type I rectal probe (EUBF334), and a $5 \mathrm{MHZ}$ convex type transrectal prostate probe

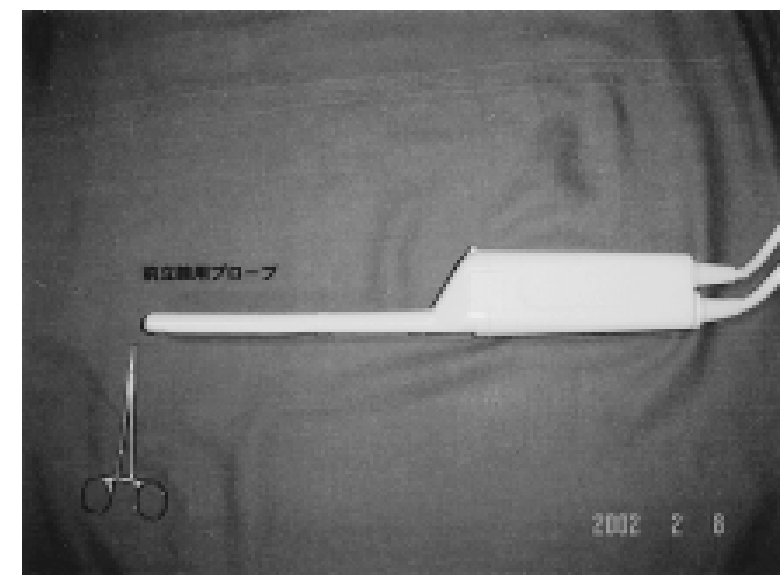

Fig. 1. Photograph of the 5MHZ convex type transrectal prostate probe (curved linear array transducer, EUP-U322, cross sectional observation field $90^{\circ}$ ), which has an approximately $18 \mathrm{~cm}$ insertion handle.

(curved linear array transducer, EUP-U322, transverse sectional observation field $90^{\circ}$ ), which have an approximately $18 \mathrm{~cm}$ insertion handle (Fig. 1).

Technique of ultrasonography: The sows were examined in breeding-sow stalls. After fecal material was manually removed from the rectum, the ovaries and uteri were examined by rectal palpation. The transrectal probe was then placed in the palm of the hand and slowly inserted into the rectum, where it was used to scan the uterus and ovaries. In gilts, it was impossible to perform rectal palpation; therefore, the transrectal prostate probe was slowly inserted into the rectum with the insertion handle, where it was used to scan the uterus.

\section{RESULTS}

Ultrasonography in sows with normal estrous cycle: Rectal palpation demonstrated that the uterus of the sows with a normal estrous cycle showed a thin pipe-like structure during the postweaning period. After the postweaning period, the tone of the uterus increased. The uterus became flaccid around the third day after ovulation. Subsequently, from the fourth day after ovulation until immediately before the recurrence of the next estrus (ovulation after 16 to 17 days), the flexibility increased with time. Transverse and sagittal sections of the uterus in the estrous and luteal phases are shown in Fig. 2. Transverse sectional ultrasonography revealed characteristic appearances of the uterus that differed between the estrous and luteal phases. The uterus in the estrous phase (Fig. 2a) showed a clear-margined echogenic thick muscle layer forming a round structure, whereas that in the luteal phase (Fig. 2c) showed an echo-genic thin muscle layer forming a comparatively wide, flattened structure, which seemed similar to the rectal palpation findings. In contrast, the sagittal section of the uterine wall in the estrous phase (Fig. 2b) appeared it show a thick muscle- 

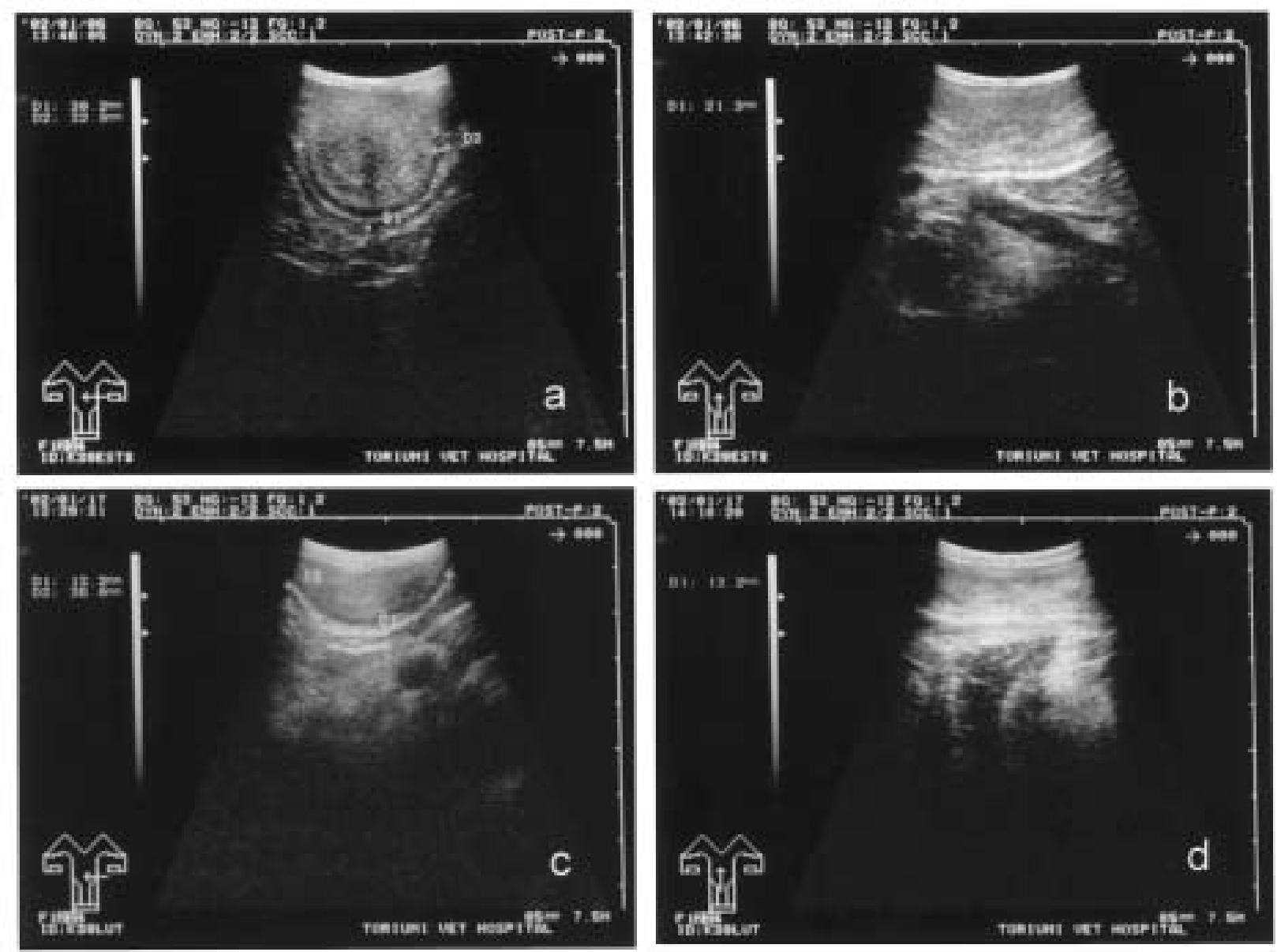

Fig. 2. Ultrasonogram of uterus in sows with normal estrous cycle. a: Transverse view of uterus in estrous phase. b: Sagittal view of uterus in estrous phase. c: Transverse view of uterus in luteal phase. d: Sagittal view of uterus in luteal phase.

layer, while the musclelayer in the luteal phase (Fig. 2d) appeared to be thin, but the difference was not conclusive. Similar findings occurred periodically in the uteri of sows with a normal estrous cycle.

Utrasonography in sows with ovarian disorders: Characteristic findings were obtained from transverse sections in the uteri of sows with OQ, OA, RCL and MLLC, as shown in Fig. 3. These findings remained the same on reexamination. The uterus of sows with OQ palpated as a slightly smaller round structure than that in the normal estrous phase. Transverse sections of the uterus of sows with OQ (Fig. 3a) showed the same findings as those in normal estrus (Fig. 2b) but with a slightly smaller size. Transverse sectional findings in the uterus of sows with OA showed an extremely thin round structure, approximately one-third of the uterine size in the estrous phase (Fig. 3b) as recognized on the rectal palpation. Transverse sectional findings in the uterus of sows with MLFC and MSFC demonstrated a clear margined echo-genic round structure similar to the uterus of the estrous phase. However, the uterus of sows with RCL and MLLC appeared as apparently different, wide, flattened structure. Ultrasonography of the uterus of sows with RCL demonstrated an echo-genic thin muscle layered over a wide, flattened structure (Fig. 3c), recognized as a flaccid balloon-like structure on rectal palpation. Transverse sections of the uterus in sows with MLLC also demonstrated an echo-genic thin muscle layer over an even wider flattened structure than that for the normal luteal phase (Fig. 3d).

Gilts: To obtain a transverse sectional observation field, a $90^{\circ}$ convex type transrectal prostate probe (EUP-U322) was used and it was possible to place the probe into the rectum without insertion of the hand. Therefore, ultrasonic image of the uterus in gilts could be obtained. The thin, round uterus of the estrous phase in gilts could be demonstrated on the ultrasonogram (Fig. 4a). In cases showing a wide, flattened uterus in the luteal phase, it was possible to obtain ultrasonographic images by scanning both sides with the probe to obtain a series of echograms. Though the probe was wide-angled, both ends of the wide, flattened uterus in the luteal phase could not be visualized on a single ultra- 

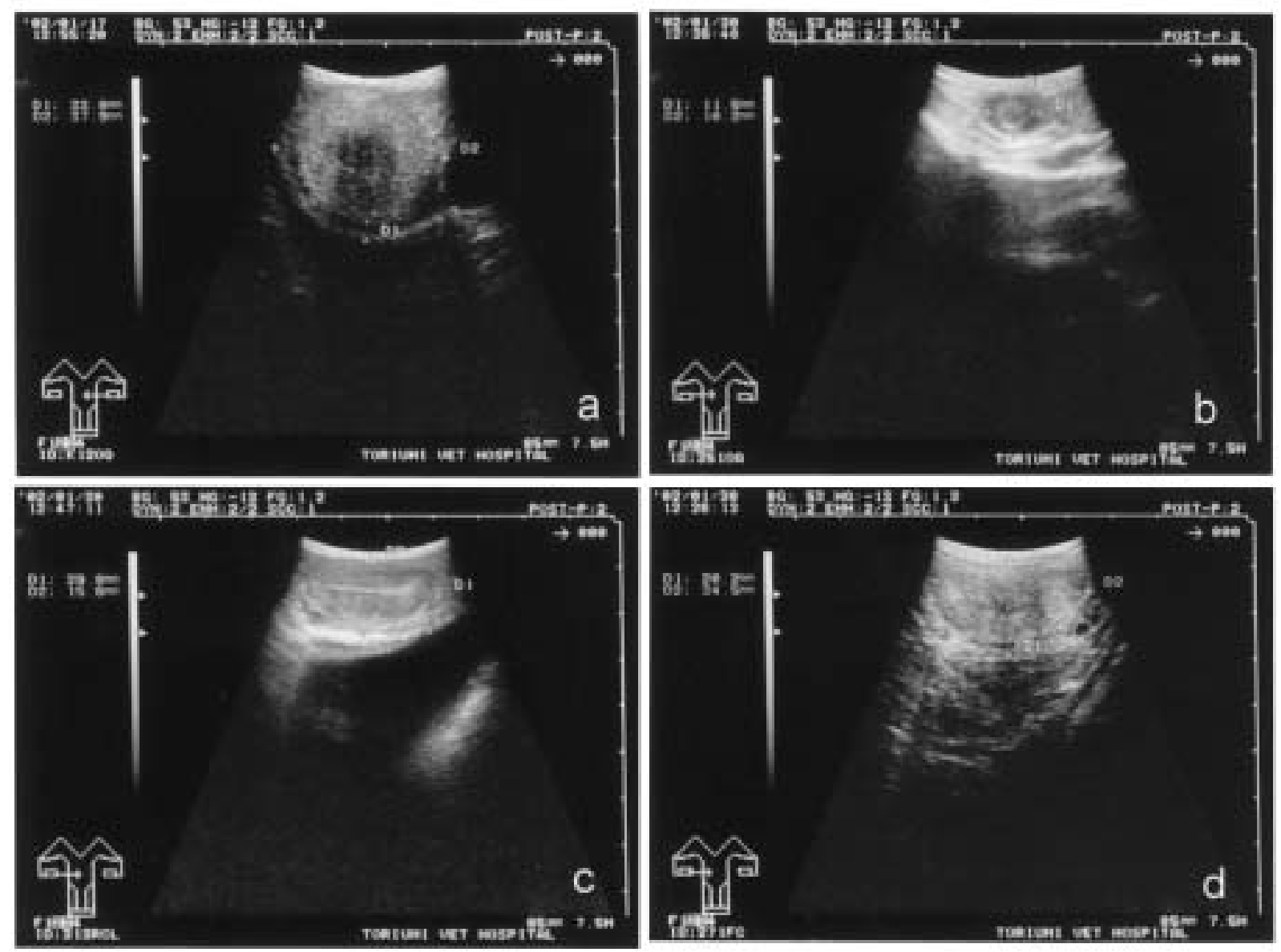

Fig. 3. Ultrasonogram of uterus in sows with ovarian disorders. a: Transverse view of uterus in sow with ovarian quiescence. b: Transverse view of uterus in sow with ovarian atrophy. c: Transverse view of uterus in sow with retention of corpus luteum.

sonogram (Fig. 4b).

\section{DISCUSSION}

In this study, the findings of ultrasonography revealed that the low $\mathrm{P}$ type uterus showed a round structure, whereas the high $\mathrm{P}$ type uterus showed a wide, flattened structure with a thin uterine wall. These results corresponded well with rectal palpation findings: low $\mathrm{P}$ type uterus was a hard, pipe-like structure and high $\mathrm{P}$ type was a soft, balloon-like structure [17]. Further, for uterine ultrasonographic diagnosis of ovarian disorders, transverse observation provided more characteristic findings than sagittal observation.

Ultrasonography using a convex type I rectal probe for multiparous sows was possible, but for gilts with a narrow pelvic (rectal) cavity, this procedure was difficult. Therefore, we employed a minimal convex type transrectal prostate probe (curved linear array transducer, EUP-U322, transverse sectional observation field $90^{\circ}$ ), which has an approximately $18 \mathrm{~cm}$ insertion handle, for gilts. The result showed that the round uterus during the estrous phase in gilts could be visualized on ultrasonograms. Although the probe was wide-angled, both ends of the particularly wide, flattened uterus were missing from the ultrasonogram. However, it was possible to observe ultrasonographic images by scanning both sides with the probe to obtain a series of echograms.

Very few studies concerning the use of ultrasonic imaging to examine normal uteri have been published. However, Kahn [7] has reported on the use of ultrasonic imaging to obtain a uniform echo pattern for a serpentine uterine horn. That study probably refered to a round uterus, but its findings differed somewhat from ours. It is likely that these discrepancies resulted from the fact that the scanners used in the two studies did not produce the same ultrasonic output.

Rectal palpation of sows is difficult compared with that of cows, and the method is not widely performed in European countries or the U.S.A. [4, 11]. Skilled practitioners are also rare in Japan. However, it must be considered that in approximately $30 \%$ of sows with postweaning anestrus, 

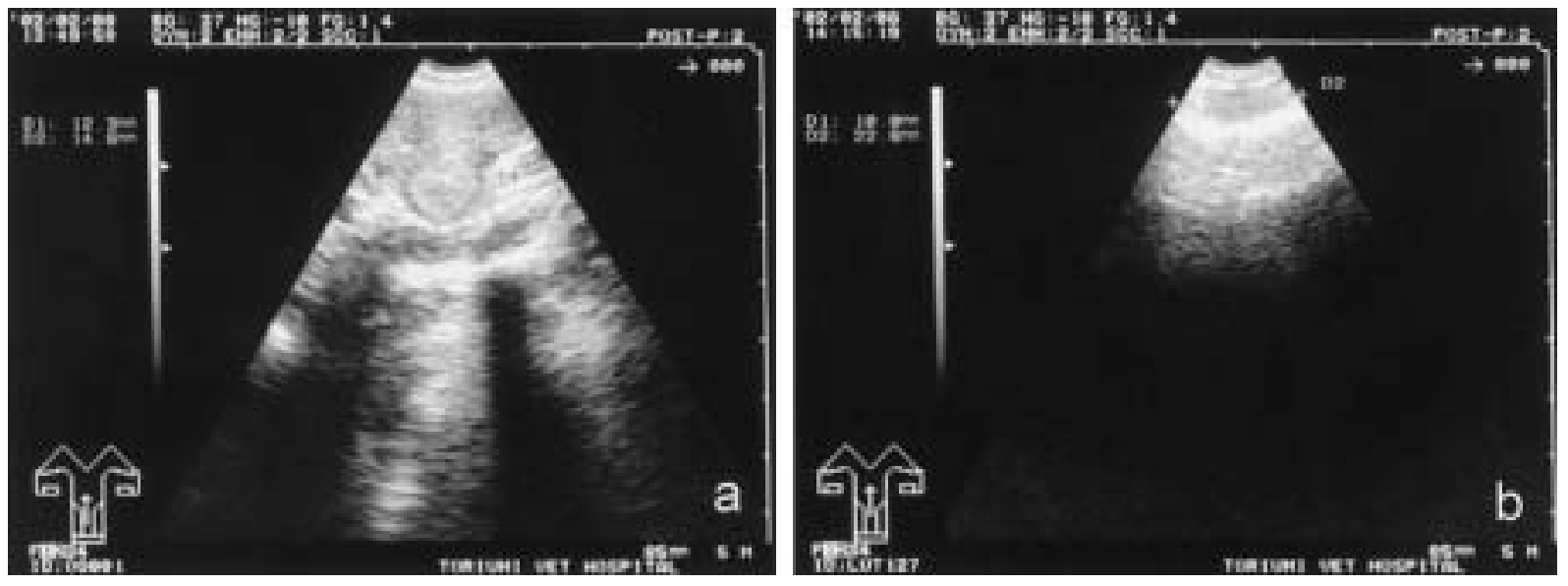

Fig. 4. Ultrasonogram of uterus in gilts in normal cycle. a: Transverse view of uterus in gilt in estrous phase. b: Transverse view of uterus in gilt in luteal phase.

the cause is retention of the corpus luteum, according to Japanese studies [16]. PMSG is routinely administered based on external clinical signs, and sows that do not respond to PMSG are culled, creating a large economic loss on the swine industry [1].

The results obtained in this study proved that uterine ultrasonography facilitated highly precise diagnosis of ovarian disorders in sows and gilts without rectal palpation. Anestrus in sows and gilts may be grossly divided into the following 2 types. Uteri demonstrating a wide, flattened shape on ultrasonography reflect ovarian disorders that actively secrete $P$. Therefore, it is possible to induce estrus after reduction of the corpus lutea by $\mathrm{PGF}_{2 \alpha}$ administration [15]. Uteri demonstrating a columnar, round shape on ultrasonography reflect ovarian disorders that do not secrete P. Therefore, PMSG or other agents are required to promote development of follicles for induction of estrus.

\section{REFERENCES}

1. Almond, G.W. 1992. Factors affecting the reproductive performance of the weaned sow. pp. 503-515. In: Vet. Clin. North Am., Food Anim. Prac., W.B. Saunders Co., Philadelphia.

2. Balke, J.M.E. and Elmore, R.G. 1982. Pregnancy diagnosis in swine. A comparison of the technique of rectal palpation and ultrasound. Theriogenology 17: 231-236.

3. Botero, O., Martinat-Botte, F. and Bariteau, F. 1986. Use of ultrasound scanning in swine for detection of pregnancy and some pathological conditions. Theriogenology 26: 267-278.

4. Cameron, R.D. 1977. Pregnancy diagnosis in sow by rectal examination. Aust. Vet. J. 53: 432-435.

5. Estill, C.T. 1997. Control of the estrous cycle in swine. pp. 703-710. In: Current Therapy in Large Animal Theriogenology (Youngquist, R.S. ed), W.B. Saunders Co., Philadelphia.

6. Irie, M., Ohmoto, K. and Kumagaya, S. 1984. Diagnosis in pigs by real time ultrasonic B-mode scan. Jpn. J. Zootech. Sci. 55: $383-388$.

7. Kahn, W. 1994. Ultrasonography in pigs, pp. 213-223. In: Veterinary Reproductive Ultrasonography (Kahn, W. ed.),
Mosby-Wolfe, London.

8. Moriyoshi, M., Sawamura, T., Yasuda, M., Nakao, T. and Kawata, K. 1996. Using ultrasound for clinical observation of the porcine ovary through the course of the estrous cycle and to monitor treatment of ovarian disease. J. Reprod. Dev. 42: 277 282.

9. National Agricultural Insurance Association (ed). 1993. pp. 76-82. In: Diagnosis and Therapy for Livestock in Japan (II). Tokyo (in Japanese).

10. Nissen, A.K., Lehn-Jensen, H., Hyttel, P. and Greve, T. 1995. Follicular development and ovulation in sows: Effect of hCG and GnRH treatment. Acta Vet. Scand. 36: 123-133.

11. Pyorala, S. 1989. Pregnancy diagnosis in swine by palpation and by amplitude-depth ultrasound scanning. Theriogenology 31: 1067-1073.

12. Ryan, D.P., Taakub, H., Harrington, D. and Lynch, P.B. 1994. Follicular development during early pregnancy and the estrous cycle of the sow. Theriogenology 42: 623-632.

13. Soeda, N.M., Noordhuizen, J.P.T.M. and Kemp, B. 1992. The duration of ovulation in pigs, studied by transrectal ultrasonography, is not related to early embryonic diversity. Theriogenology 38: 653-666.

14. Toriumi, H., Kuwahara, Y., Ichikawa, Y., Takagi, K., Tsumagari, S. and Takeishi, M. 2000. Uterine contraction in sows during estrus and puerperium, and in sows with multiple follicular cysts. J. Reprod. Dev. 46: 9-14

15. Toriumi, H., Kuwahara, Y., Ichikawa, Y., Shimizu, K., Tsumagari, S., Takeishi, M. and Xie, H. 2001. Comparative study on the effectiveness of aquapuncture and intramuscular injection of fenprostalen, dinoprost and cloprostenol on retention of the corpus luteum in sows. J. Am. Holist. Vet. Med. Assoc 20: 913.

16. Toriumi, H., Tsumagari, S., Kuwahara, Y., Takagi, K., Ohba, S., Shimizu, K. and Takeishi, M. 1995. Clinical findings and serum steroid hormone concentrations in sows with post-weaning ovarian disorders. p. 147. Proceeding of 25th Congress of the World Veterinary Association.

17. Toriumi, H., Tsumagari, S., Kuwahara, Y., Takagi, K., Ohba, S. and Takeishi, M. 1996. Clinical findings and serum steroid hormone concentrations in sows with post-weaning ovarian disorders. J. Reprod. Dev. 42: 1-6. 